Stacking and electric field effects in atomically thin layers of GaN

This content has been downloaded from IOPscience. Please scroll down to see the full text. 2013 J. Phys.: Condens. Matter 25345302

(http://iopscience.iop.org/0953-8984/25/34/345302)

View the table of contents for this issue, or go to the journal homepage for more

Download details:

IP Address: 128.173.176.52

This content was downloaded on 25/09/2013 at 19:53

Please note that terms and conditions apply. 


\title{
Stacking and electric field effects in atomically thin layers of GaN
}

\author{
Dongwei Xu ${ }^{1}$, Haiying $\mathrm{He}^{1}$, Ravindra Pandey ${ }^{1}$ and Shashi P Karna ${ }^{2}$ \\ ${ }^{1}$ Department of Physics, Michigan Technological University, Houghton, MI 49931, USA \\ ${ }^{2}$ US Army Research Laboratory, Weapons and Materials Research Directorate, ATTN: RDRL-WM, \\ Aberdeen Proving Ground, MD 21005-5069, USA \\ E-mail: hhe@mtu.edu and pandey@mtu.edu
}

Received 30 May 2013, in final form 11 July 2013

Published 29 July 2013

Online at stacks.iop.org/JPhysCM/25/345302

\begin{abstract}
Atomically thin layers of nitrides are a subject of interest due to their novel applications. In this paper, we focus on $\mathrm{GaN}$ multilayers, investigating their stability and the effects of stacking and electric fields on their electronic properties in the framework of density functional theory. Both bilayers and trilayers prefer a planar configuration rather than a buckled bulk-like configuration. The application of an external perpendicular electric field induces distinct stacking-dependent features in the electronic properties of nitride multilayers: the band gap of a monolayer does not change whereas that of a trilayer is significantly reduced. Such a stacking-dependent tunability of the band gap in the presence of an applied field suggests that multilayer $\mathrm{GaN}$ is a good candidate material for next generation devices at the nanoscale.
\end{abstract}

S] Online supplementary data available from stacks.iop.org/JPhysCM/25/345302/mmedia

(Some figures may appear in colour only in the online journal)

\section{Introduction}

Thin layers of semiconducting materials with thickness less than $100 \mathrm{~nm}$ are reshaping the design paradigms of electronics and optoelectronics devices [1-3]. The reduced dimensionality, prevailing quantum confinement, and extremely high specific surface ratios and strain modulation in these ultrathin films lend them new and novel physical properties. In this regard, $\mathrm{GaN}$ is of particular interest, since the bulk GaN has unique optoelectronic properties, high mechanical stability and good thermal conductivity [4-6]. A relatively large and direct band gap [7] of about $3.4 \mathrm{eV}$ also makes $\mathrm{GaN}$ an excellent host material for light-emitting devices (LEDs) that operate in the blue and ultraviolet region [8]. Note that the traditional high-brightness LEDs consisted of a thin epitaxial film of $\mathrm{GaN}$ deposited on sapphire, which is expensive. However, recent breakthroughs have been made [9] in growing epitaxial $\mathrm{GaN}$ on $\mathrm{Si}$, making this material economically competitive with other low-cost materials. There have also been recent advances in the synthesis and fabrication of GaN with nanometer-scale thickness [10-13], which hold promise for the realization of a nanoscale device composed of a few atomic layers of $\mathrm{GaN}$ in the near future.

Key to the application of an atomically thin multilayer $\mathrm{GaN}$ in next generation optoelectronic devices is the ability to tailor and tune its band gap at the nanoscale. This is what we intend to address in this paper by taking a bottom-up approach to represent the ultimate configuration of an ultrathin film of $\mathrm{GaN}$ by a two-dimensional (2D) atomic layer. Specifically, we consider bi-, and tri-layers of $\mathrm{GaN}$ in different stacking geometries and investigate their equilibrium structure, band structure, atomic projected density of states without and in the presence of an externally applied electric field using the first-principles density functional method. The geometrical and electronic structures of monolayer GaN without and in the presence of an external electric field have been recently investigated [14, 15]. Similar studies on stacked 2D planar nanostructures, such as graphene, BN, and $\mathrm{BN}$-graphene hybrid [16-21] using theoretical techniques of varying sophistication, have revealed a number of important features, including tenability of band gap by electric field in some configurations. 

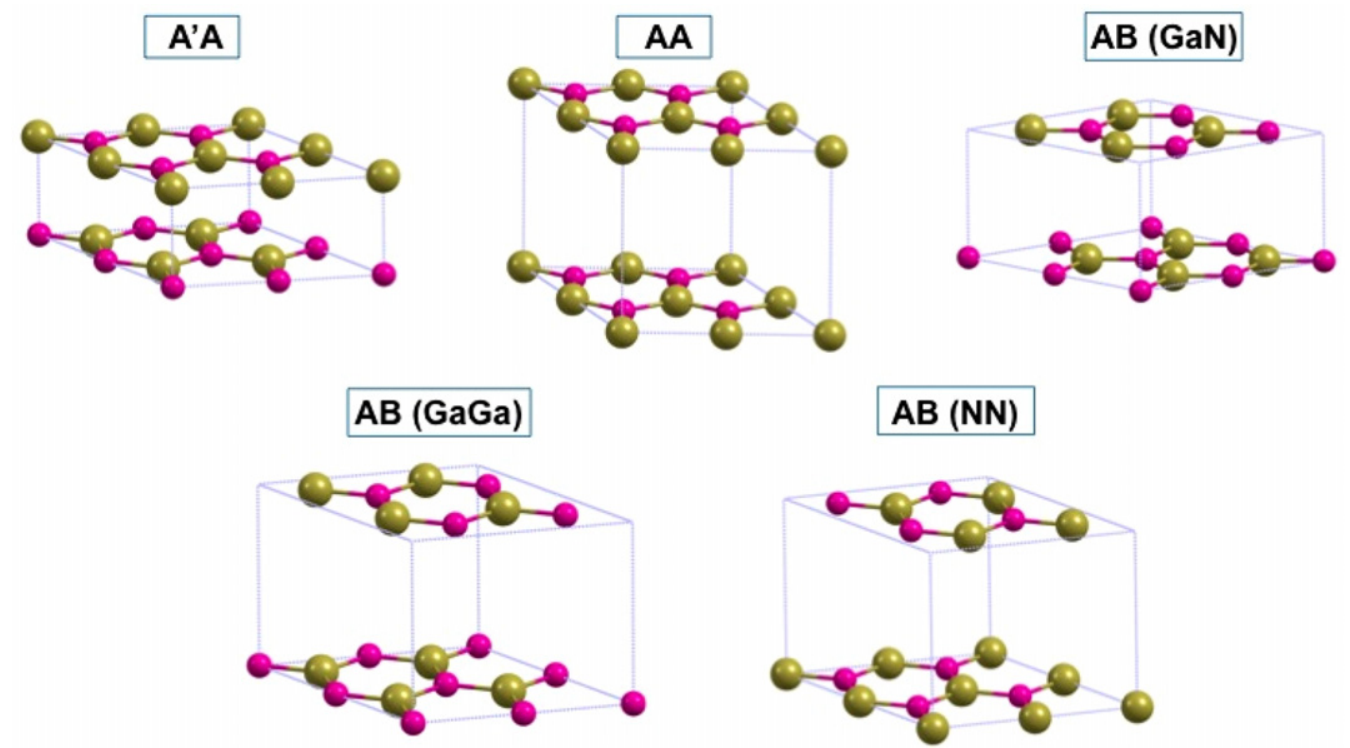

Figure 1. Stacking configurations considered for the bilayer of GaN. The large brown balls represent 'Ga' while the small pink balls represent ' $\mathrm{N}$ '.

The computational details and validation of our method are given in section 2 . In section 3.1, we present the results of structural evolution of the multilayer $\mathrm{GaN}$ in going from a single layer to three layers. The stacking alternatives of the multilayer GaN are fully explored. The electronic properties and their dependence on the stacking sequence are presented in section 3.2. In section 3.3, the response of a multilayer GaN under an external perpendicular electric field is reported. A brief summary of the results is given in section 4 .

\section{Method}

The projector augmented plane-wave (PAW) method as implemented in the Vienna $a b$ initio simulation package (VASP) is employed [22, 23]. The generalized gradient approximation (GGA) of Perdew, Burke and Ernzerhof (PBE) is adopted for the exchange-correlation functional [24] to the density functional theory (DFT), together with the van der Waals (vdW) interactions described via a pair-wise force field in the DFT-D2 method of Grimme [25]. The 2s and $2 p$ electrons of nitrogen, $3 d, 4 s$ and $4 p$ electrons of $\mathrm{Ga}$ are considered explicitly as the valance electrons for the electronic structure calculations.

A minimum vacuum distance of $12 \AA$ between neighboring images is used in the supercell employed. The cut-off energy for the plane-wave basis set is set to $520 \mathrm{eV}$. The energy tolerance is $10^{-6} \mathrm{eV}$ in the iterative solution of the Kohn-Sham equations. The structure and atoms are relaxed until the force on each atom is less than $0.01 \mathrm{eV} \AA^{-1}$. The Monkhorst-Pack mesh of $(15 \times 15 \times 1)$ is selected in the Brillouin-zone integrations for the structural optimization and density of state (DOS) calculations. It should be pointed out that the GGA-DFT method has been shown to provide a reasonably good description of the physics and chemistry of GaN systems [26, 27] although it underestimates its band gap.

In order to validate our modeling elements, the calculated structure and electronic properties of the most common wurtzite structure of the bulk GaN are compared with the experimental and previously reported theoretical studies. The calculated lattice constants, $a=3.204 \AA, c=5.240 \AA$ at the vdW-DFT level of theory are in good agreement with the corresponding experimental values of $a=3.185 \AA, c=$ $5.185 \AA[7,28]$. The vdW-DFT band gap is $1.74 \mathrm{eV}$, which is in line with the previous DFT calculations [29], but lower than the experimental value of $3.4 \mathrm{eV}$ [7].

\section{Results and discussion}

\subsection{Geometry}

A single layer of GaN perpendicular to the (001) direction is cleaved from the wurtzite $\mathrm{GaN}$, and fully relaxed during the geometry optimization. Unlike the bulk wurtzite structure where each atom is four-fold coordinated, the monolayer GaN takes a planar graphene-like structure with three-fold coordination in its ground state. The calculated $\mathrm{Ga}-\mathrm{N}$ bond length is $1.85 \AA$, which is smaller than the bulk value of $1.96 \AA$. Note the bond lengths calculated at either the GGA-DFT or vdW-DFT level of theory are the same, suggesting the presence of the predominant semi-ionic bonding in a single atomic layer as the case with the bulk GaN. These results are comparable with our previous results [15] obtained at the GGA-DFT level of theory using the SIESTA computer code, where the bond length is reported to be $1.91 \AA$ for the monolayer $\mathrm{GaN}$.

By bringing two single layers together, one can form a bilayer. There exist five possible stacking sequences for such a bilayer system, i.e., AA, $\mathrm{AA}^{\prime}, \mathrm{AB}(\mathrm{NN}), \mathrm{AB}(\mathrm{GaGa})$, $\mathrm{AB}(\mathrm{NGa})$, as shown in figure 1. Similar to a hexagonal monolayer of $\mathrm{BN}$, the monolayer of $\mathrm{GaN}$ can be divided into two sublattices: Ga atoms occupying one sublattice and $\mathrm{N}$ atoms occupying the other. In a bilayer, we label the atoms of the bottom and top layers as $\mathrm{X} 1(\mathrm{X}=\mathrm{N}$ or Ga) and 

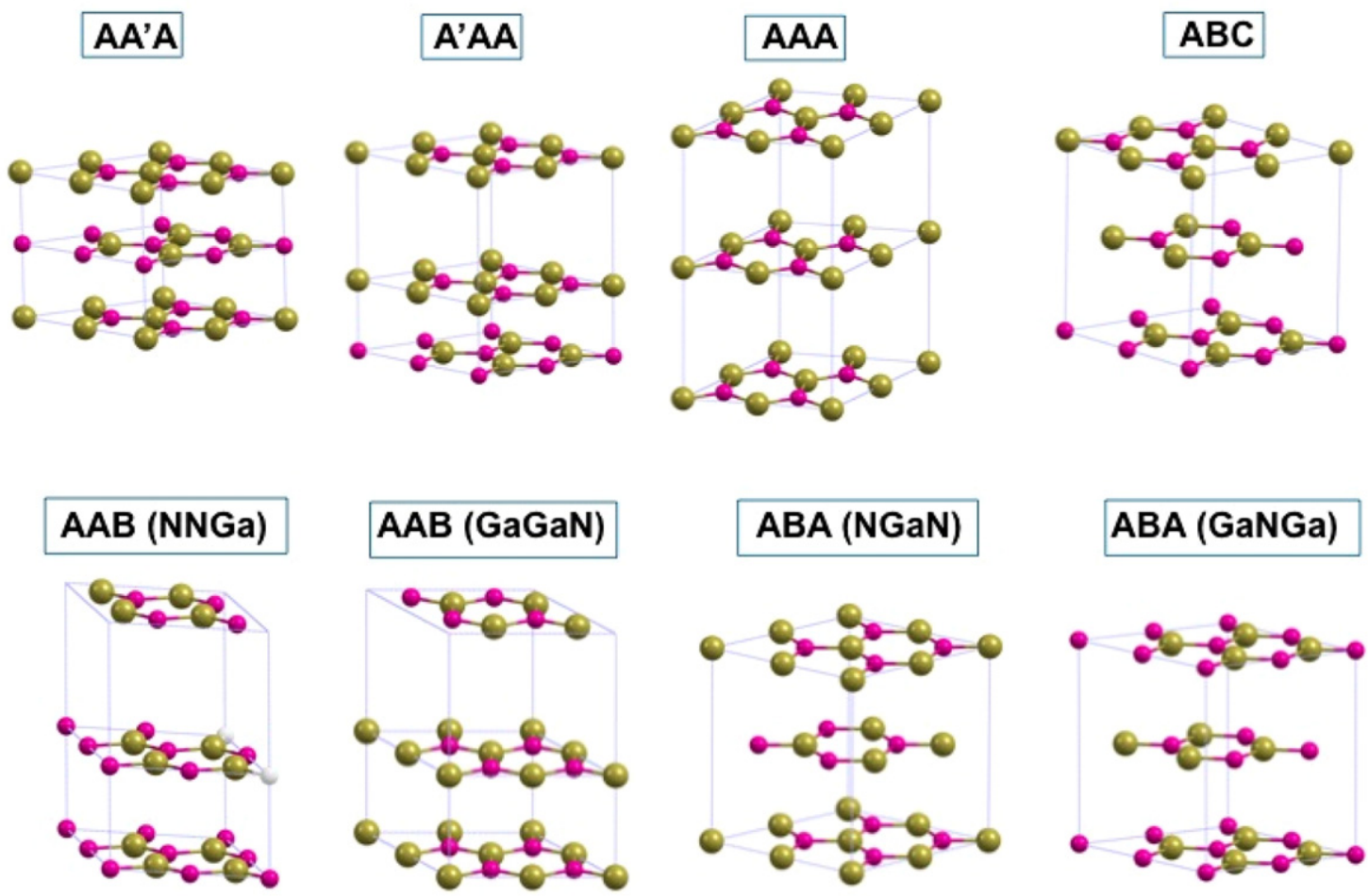

Figure 2. Stacking configurations considered for the trilayer of GaN. The large brown balls represent 'Ga' while the small pink balls represent ' $\mathrm{N}$ '.

$\mathrm{X} 2(\mathrm{X}=\mathrm{N}$ or $\mathrm{Ga})$, respectively. For the $\mathrm{AA}^{\prime}$ (hexagonal) stacking, the sublattice $\mathrm{N} 2(\mathrm{Ga} 2)$ is located directly on top of Ga1 (N1). We have also considered the AA stacking, where the sublattice $\mathrm{N} 2(\mathrm{Ga} 2)$ is located directly on top of N1 (Ga1). For the AB (Bernal-type) stacking, only one sublattice of the top layer is located on the top of a sublattice of the bottom layer and the other sublattice is on top of the center of the hexagon of the bottom layer. If the atoms on top of each other are $\mathrm{N} 2$ and $\mathrm{N} 1$, it is designated as $\mathrm{AB}(\mathrm{NN})$ stacking. If they are $\mathrm{Ga} 2$ and $\mathrm{Ga} 1$, it is labeled as $\mathrm{AB}(\mathrm{GaGa})$ stacking. If they are of different species, it is designated as $A B(\mathrm{GaN})$, which is essentially the same as $\mathrm{AB}$ (NGa) (figure 1).

By adding one more layer to a bilayer, a trilayer is formed, for which, as shown in figure 2, eight stacking sequences are considered. The trilayers are constructed in such a way that the stacking order of the neighboring two layers follows one of the $\mathrm{AA}, \mathrm{AA}^{\prime}$ and $\mathrm{AB}(\mathrm{NGa})$ stackings of the bilayers. For instance, $\mathrm{AA}^{\prime}$ stacking of the lower two layers and $\mathrm{A}^{\prime} \mathrm{A}$ stacking of the top two layers form the $\mathrm{AA}^{\prime}$ A stacked trilayer. Similarly, AA + AA form AAA, $\mathrm{AA}+\mathrm{AB}$ form $\mathrm{AAB}(\mathrm{NNGa})$ or $\mathrm{AAB}(\mathrm{GaGaN})$ depending on the relative shift of the top layer. $\mathrm{A}^{\prime} \mathrm{A}+\mathrm{AA}$ can form $\mathrm{A}^{\prime} \mathrm{AA}$, and $\mathrm{AB}+\mathrm{AB}$ can form three different stacked configurations for the trilayer configuration.

The optimized bond lengths, interlayer distances, cohesive energies $\left(E_{\mathrm{coh}}\right)$ of the bi- and trilayer configurations of $\mathrm{GaN}$ are listed in table 1 . The cohesive energy is defined as

$$
E_{\mathrm{coh}}=\left(\sum_{i}^{N_{\text {pair }}}\left(E_{\mathrm{N}}+E_{\mathrm{Ga}}\right)-E_{\mathrm{total}}\right) / N_{\text {pair }},
$$

where, $E_{\mathrm{N}}$ and $E_{\mathrm{Ga}}$ are the energies of the isolated atom $\mathrm{N}$ and Ga, respectively, and $N_{\text {pair }}$ is the number of $\mathrm{N}-\mathrm{Ga}$ pairs in calculating the total energy of the system. This intrinsic parameter reflects the stability of the multilayer systems. In the following, we will primarily use the vdW-DFT results for discussion. A comparison of the vdW-DFT results with the GGA-DFT results will be given at the end of this section.

A cohesive energy of $8.14 / 8.38 \mathrm{eV}$ is computed for the GaN monolayer at the GGA-DFT/vdW-DFT level of theory. It agrees fairly well with the previous studies using the GGA functional forms $[14,15]$, but deviates significantly from the reported results using the LDA functional forms [30, 31]. For the bilayer $\mathrm{GaN}$, we find that the hexagonal $\mathrm{AA}^{\prime}$ stacking is the most energetically favorable $\left(E_{\text {coh }}=8.68 \mathrm{eV} / \mathrm{Ga}-\mathrm{N}\right.$ pair), followed by the Bernal-type $\mathrm{AB}(\mathrm{GaN})$ stacking $\left(E_{\mathrm{coh}}=\right.$ $8.58 \mathrm{eV} / \mathrm{Ga}-\mathrm{N}$ pair). The $\mathrm{AA}, \mathrm{AB}(\mathrm{NN})$ and $\mathrm{AB}(\mathrm{GaGa})$ stackings are found to be relatively less stable (table 1). The order of stability is also reflected in the calculated interlayer binding energy $\left(0.58 \mathrm{eV}\right.$ and $0.41 \mathrm{eV}$ for $\mathrm{AA}^{\prime}$ and $\mathrm{AB}(\mathrm{GaN})$ stackings, respectively) and the interlayer separation $(2.47 \AA$ and $2.94 \AA$ for $\mathrm{AA}^{\prime}$ and $\mathrm{AB}(\mathrm{GaN})$ stackings, respectively). The smaller the interlayer separation, the higher the binding energy is.

The magnitude of the interlayer binding energy for different stackings can be traced back to the contributions of the electrostatic and vdW interactions. In the semi-ionic GaN lattice, the cation (gallium) tends to attract an anion (nitrogen), and repel another cation. Thus, in the $\mathrm{AA}^{\prime}$ stacking, each $\mathrm{Ga}(\mathrm{N})$ sublattice of one layer tends to attract the adjacent $\mathrm{N}$ (Ga) sublattice of the other layer, resulting in a small interlayer spacing and a higher interlayer binding energy. 
Table 1. Intraplanar bond length $R_{\text {bond }}$, interplanar separation $R_{\text {interlayer }}$, and cohesive energy $E_{\text {coh }}$ for the monolayer, bilayer and trilayer GaN after full optimization by GGA-DFT and GGA + vdW-DFT methods. In the third column showing the corresponding figures, the small purple ball represents ' $\mathrm{N}$ ' and the large brown ball represents ' $\mathrm{Ga}$ '.

\begin{tabular}{|c|c|c|c|c|c|c|}
\hline & Stackings & Representative figure & Method & $R_{\text {bond }}(\AA)$ & $R_{\text {interlayer }}(\AA)$ & $E_{\text {coh }}(\mathrm{eV} /$ pair $)$ \\
\hline \multirow[t]{2}{*}{ Monolayer } & & & GGA-DFT & 1.85 & - & 8.14 \\
\hline & & & vdW-DFT & 1.85 & - & 8.38 \\
\hline \multirow[t]{4}{*}{ Biayer (hexagonal type) } & $\mathrm{AA}$ & & GGA-DFT & 1.85 & 4.66 & 8.14 \\
\hline & & & vdW-DFT & 1.85 & 3.63 & 8.46 \\
\hline & $\mathrm{AA}^{\prime}$ & & GGA-DFT & 1.89 & 2.40 & 8.14 \\
\hline & & & vdW-DFT & 1.88 & 2.47 & 8.68 \\
\hline \multirow[t]{6}{*}{ Bilayer (Bernal type) } & $\mathrm{AB}(\mathrm{GaN})$ & & GGA-DFT & 1.85 & 3.30 & 8.16 \\
\hline & & & vdW-DFT & 1.86 & 2.94 & 8.58 \\
\hline & $\mathrm{AB}(\mathrm{NN})$ & & GGA-DFT & 1.85 & 4.54 & 8.14 \\
\hline & & & vdW-DFT & 1.85 & 3.46 & 8.46 \\
\hline & $\mathrm{AB}(\mathrm{GaGa})$ & & GGA-DFT & 1.85 & 4.33 & 8.17 \\
\hline & & & vdW-DFT & 1.85 & 3.38 & 8.48 \\
\hline \multirow[t]{6}{*}{ Trilayer (hexagonal type) } & AAA & & GGA-DFT & 1.85 & 4.66 & 8.14 \\
\hline & & & vdW-DFT & 1.85 & 3.64 & 8.48 \\
\hline & $\mathrm{AA}^{\prime} \mathrm{A}$ & & GGA-DFT & 1.89 & 2.45 & 8.30 \\
\hline & & & vdW-DFT & 1.89 & 2.49 & 8.78 \\
\hline & $\mathrm{A}^{\prime} \mathrm{AA}$ & & GGA-DFT & 1.87 & $2.49,4.46^{\mathrm{a}}$ & 8.20 \\
\hline & & & vdW-DFT & 1.87 & $2.54,3.59$ & 8.62 \\
\hline \multirow[t]{2}{*}{ Trilayer (Bernal type) } & $\mathrm{ABA}(\mathrm{NGaN})$ & & GGA-DFT & 1.86 & 3.32 & 8.16 \\
\hline & & & vdW-DFT & 1.86 & 2.94 & 8.66 \\
\hline \multirow[t]{2}{*}{ Trilayer (rhombohedral) } & $\mathrm{ABC}$ & & GGA-DFT & 1.86 & 3.28 & 8.16 \\
\hline & & & vdW-DFT & 1.86 & 2.91 & 8.66 \\
\hline
\end{tabular}

a If two numbers are given in one cell, they correspond to the interlayer distance between the bottom two layers and between the top two layers respectively.

In the AA stacking, each Ga $(\mathrm{N})$ sublattice of one layer tends to repel the adjacent $\mathrm{Ga}(\mathrm{N})$ sublattice of the other layer, resulting in a large interlayer spacing and barely any interlayer binding energy. Very strikingly, the effect of vdW interactions is not negligible in this case and the interlayer spacing reduces from 4.66 to $3.63 \AA$ after including the vdW terms in DFT. Nonetheless, the intralayer bond length gets stretched slightly with respect to that of a monolayer $(\approx 1.85 \AA)$ at the vdW-DFT level of theory.

For trilayers, $\mathrm{AA}^{\prime} \mathrm{A}$ is found to be the most stable stacking configuration, and is followed by $\mathrm{A}^{\prime} \mathrm{AA}, \mathrm{ABA}$ (or $\mathrm{ABC}$ ), $\mathrm{AAB}$ and AAA stackings. The stability of the $\mathrm{AA}^{\prime} \mathrm{A}$ stacked trilayer can be attributed to the alternating cation-anion arrangements between the layers (figure 2). The interlayer binding energy in this case is $1.20 \mathrm{eV}$, the highest of all. As a result, the $\mathrm{AA}^{\prime} \mathrm{A}$ stacking has the smallest interlayer distance and largest intralayer bond length amongst the trilayer configurations considered. Note that there exist two values for the interlayer spacing for $\mathrm{A}^{\prime} \mathrm{AA}$ because of the different stacking order between the first and the second layer, and between the second and the third layer (table 1).

Our results therefore find the most stable stacking sequence of $\mathrm{GaN}$ atomic layers to be $\mathrm{AA}^{\prime} \mathrm{AA}^{\prime} \ldots$. The calculated cohesive energy increases with the number of layers; monolayer (8.38 eV/Ga-N pair), the $\mathrm{AA}^{\prime}$ bilayer (8.68 eV/Ga-N pair), and the $\mathrm{AA}^{\prime}$ A trilayer $(8.78 \mathrm{eV} / \mathrm{Ga}-\mathrm{N}$ pair) indicating the increasing stability of the multilayer system with increasing the number of layers and approaching 
the value of wurtzite $\mathrm{GaN}$ bulk (9.74 eV/Ga-N pair). Our calculated bulk cohesive energy at the GGA-vdW-DFT level of theory agrees well with previous GGA results (9.265 eV/Ga-N pair), while significantly lower than the LDA results (10.999 $\mathrm{eV} / \mathrm{Ga}-\mathrm{N}$ pair) owing to the fact that LDA overestimates binding energies [32]. Thus, the hexagonal stacked configuration is predicted to be the most stable configuration for the multilayer $\mathrm{GaN}$. This is in contrast to the case of the bilayers of graphene [33] and BN [34] where the Bernal stacking forms the ground state. On the other hand, both Bernal and rhombohedral stackings are stable for the trilayer graphene [35-37]. We attribute the difference in graphene, $\mathrm{BN}$ and $\mathrm{GaN}$ to their ionicity. A larger degree of ionicity renders the $\mathrm{AA}^{\prime}$ and $\mathrm{AA}^{\prime} \mathrm{A}$ stackings to be the more stable configurations for $\mathrm{GaN}$.

It is interesting to see a similarity in the stacking sequence shared by the multilayer $\mathrm{GaN}$ and the bulk GaN, though the layers are buckled in the bulk. Nonetheless, it is worth noting that the planar structure is always the preferred structure for both the bilayer and trilayer configurations of GaN. There may exist a small buckling in which a maximum variation in the interlayer spacing is less than $1 \%$. The predicted preference of planar configuration is further verified by taking a buckled bilayer directly cut from the bulk wurtzite $\mathrm{GaN}$ as the initial configuration for the unconstrained geometry optimization. The buckled bilayer relaxes to a nearly planar configuration in which the buckling is reduced from $0.67 \AA$ to about $0.067 \AA$. This is different from what has been reported for the GaN nanoribbons [38] where the bilayer nanoribbons cleaved from the bulk $\mathrm{GaN}$ are predicted to be more stable as compared to the planar nanoribbons. We believe that the difference comes from the periodic 2D sheet (our model) and 2D nanoribbon with edges [38]. The existence of edges may be the cause of the buckling in nanoribbons. We note that the preference of the planar configuration is consistent with other theoretical studies on the GaN sheet [31]. Furthermore, research on the structural evolution of two-dimensional films has shown that the system prefers a planar configuration when the number of layers is less than six [39].

Finally, our calculations also demonstrate the importance of the vdW terms for the layered structures, even in a semi-ionic material, like GaN. Table 1 gives the results at vdW-DFT and GGA-DFT level of theory showing the same order of the stacking-dependent stability for the multilayer GaN. A comparison of the structural properties suggests that the vdW terms do not change the intraplanar bond lengths, though it influences significantly the interplanar separations. The effect is more prominent for the cases where there is no bonding or barely any electrostatic interactions between layers, such as the $\mathrm{AA}, \mathrm{AB}(\mathrm{NN})$ and $\mathrm{AB}(\mathrm{GaGa})$ bilayers (table 1). Inclusion of the vdW interaction terms drastically reduces the optimal interlayer distance by more than $1 \AA$ with an increase in the interlayer binding of about $0.2 \mathrm{eV}$. Note that Marom et al have also pointed out the significant role of the $\mathrm{vdW}$ forces in anchoring the layers at a fixed distance for the BN layered structures [40].

\subsection{Electronic structure}

We now consider the configurations obtained at the vdW-DFT level of theory for calculations of electronic properties of the multilayer GaN. The calculated band structures of monolayer, bilayer and trilayer configurations are shown in figure 3 (solid lines).

The monolayer has an indirect band gap of $2.17 \mathrm{eV}$. The conduction band minimum $(\mathrm{CBM})$ is located at $\Gamma$ whereas the valence band maximum (VBM) is located at $\mathrm{K}$. A higher value of the band gap for the monolayer relative to that of the bulk wurtzite $\mathrm{GaN}$ can be attributed to the quantum confinement effect [15]. Similar to the monolayer, an indirect band gap with the values of 2.01 and $1.69 \mathrm{eV}$ for the $\mathrm{AA}^{\prime}$ and $\mathrm{AB}(\mathrm{GaN})$ stacked configurations, respectively is predicted. For the $A B$ $(\mathrm{GaN})$ bilayer, lowering of the symmetry breaks degeneracy between the top two valance bands at K. For the $\mathrm{AA}^{\prime} \mathrm{A}$ and ABA (NGaN) stacked trilayers, the band gap is indirect with the values of 1.85 and $1.60 \mathrm{eV}$, respectively.

A clear trend of change in electronic properties with the number of layers is noted. The bulk GaN can be deemed as an $\mathrm{AA}^{\prime}$-stacked (buckled) configuration with infinite number of layers. It has a Ga-N bond length of $1.97 \AA$ and a calculated band gap of $1.74 \mathrm{eV}$. The $\mathrm{AA}^{\prime} \mathrm{A}$-stacked trilayer has an intralayer Ga-N bond length of $1.89 \AA$ and a gap of $1.85 \mathrm{eV}$. The $\mathrm{AA}^{\prime}$-stacked bilayer has an intralayer bond length of $1.88 \AA$ and a gap of $2.01 \mathrm{eV}$. Finally, the monolayer has a bond length of $1.85 \AA$ and a gap of $2.17 \mathrm{eV}$. The calculated results therefore show that the decrease in the intralayer bond length is accompanied by an increase in the band gap as the interlayer interaction is gradually weakened in the multilayer $\mathrm{GaN}$.

In order to understand the predicted variation of the band gap as a function of the number of layers, the atom-resolved projected density of states (PDOS) for monolayer, the $\mathrm{AA}^{\prime}$ bilayer and $\mathrm{AA}^{\prime} \mathrm{A}$ trilayer configurations are shown in figure 4 . In all cases, the top of the valance band is formed by the nitrogen atoms. For the $\mathrm{AA}^{\prime}$-stacked bilayer, the top and bottom layers have a point symmetry. As a result, we find the degeneracy between the energy spectrum of the $\mathrm{Ga}(\mathrm{N})$ atoms of the top layer and that of the $\mathrm{Ga}(\mathrm{N})$ atoms of the bottom layer. The interlayer interaction appears to increase the width of the valence band from $6.06 \mathrm{eV}$ for the monolayer to $6.45 \mathrm{eV}$ for the bilayer. The conduction band states associated with $\mathrm{Ga}$ and $\mathrm{N}$ atoms are also broadened reflecting the presence of the interlayer interaction in the bilayer GaN.

There exists an intimate relationship between the band structure and the inter-atomic interactions in these atomic layered structures as explained by a simple orbital interaction model (OIM) (figure 5) showing the shift of energy levels as a result of the interlayer interaction. The valence electrons are only considered, and the energy levels are referred to the average value of the corresponding energy spectra. Due to the different electronegativity, the energy levels $(4 s 4 p)$ of $\mathrm{Ga}$ are higher than those of $\mathrm{N}$ (2s2p). For simplicity, one representative level is shown for each case in the energy diagram ignoring the hybridization of $\mathrm{Ga}$ and $\mathrm{N}$ orbitals. In a monolayer, the energy level of Ga shifts up and forms the 

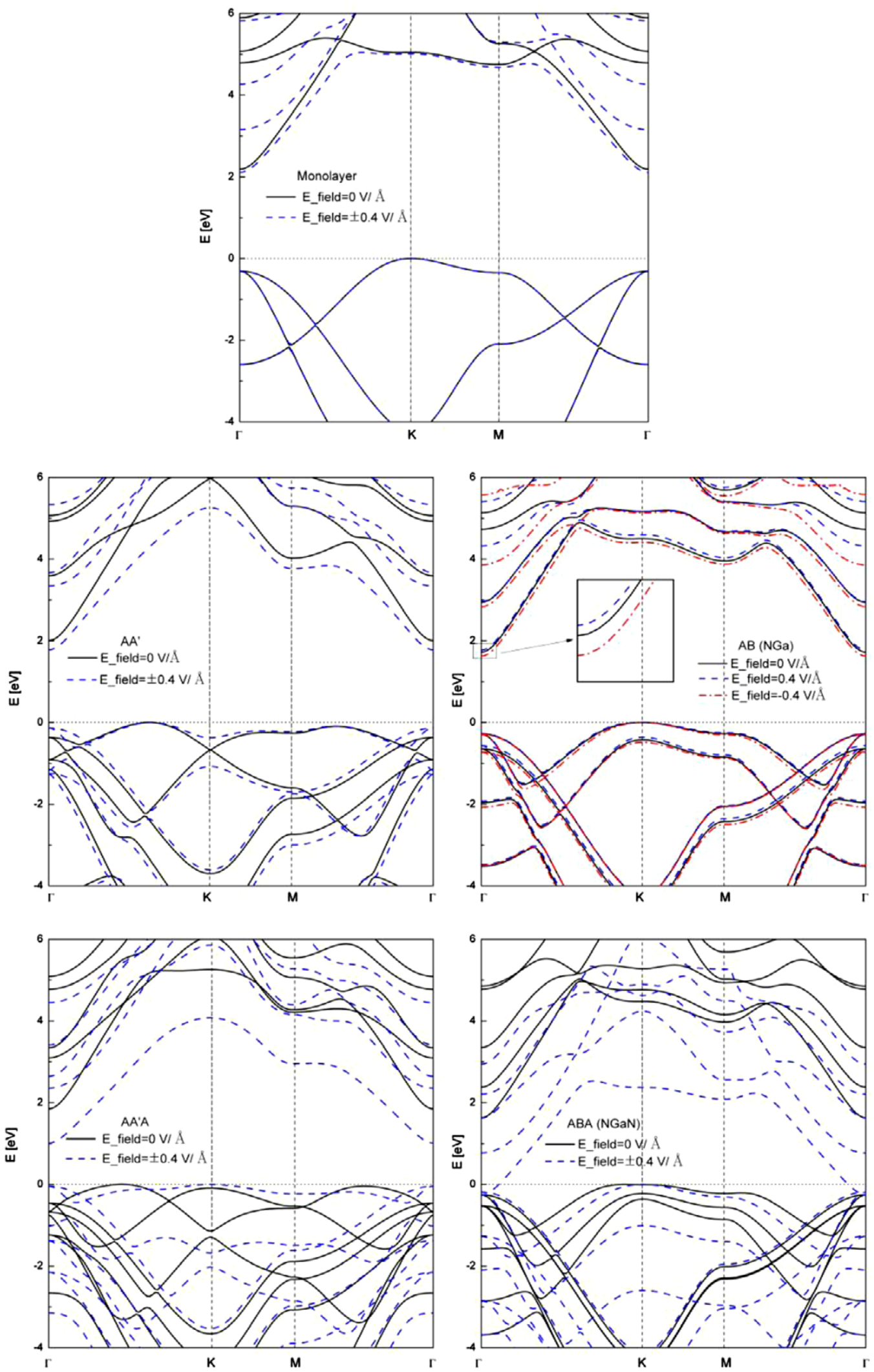

Figure 3. The calculated band structures of the monolayer (upper panes), the bilayers (middle panes) and the trilayers (bottom panes) of $\mathrm{GaN}$ with hexagonal and Bernal stackings. Solid (black), dashed (blue) and dot-dashed (red) lines represent the applied field of $0 \mathrm{~V} \AA^{-1}, 0.4 \mathrm{~V} \AA^{-1}$ and $-0.4 \mathrm{~V} \AA^{-1}$, respectively. The positive direction of field is defined from bottom to top for all the structures (figures 1 and 2). Except for the case of $\mathrm{AB}(\mathrm{NGa})$, the band structures under positive and negative fields are the same due to the symmetry. The inset shows the zoom-in near the conductance band minimum for the $\mathrm{AB}$ stacked $\mathrm{GaN}$. 

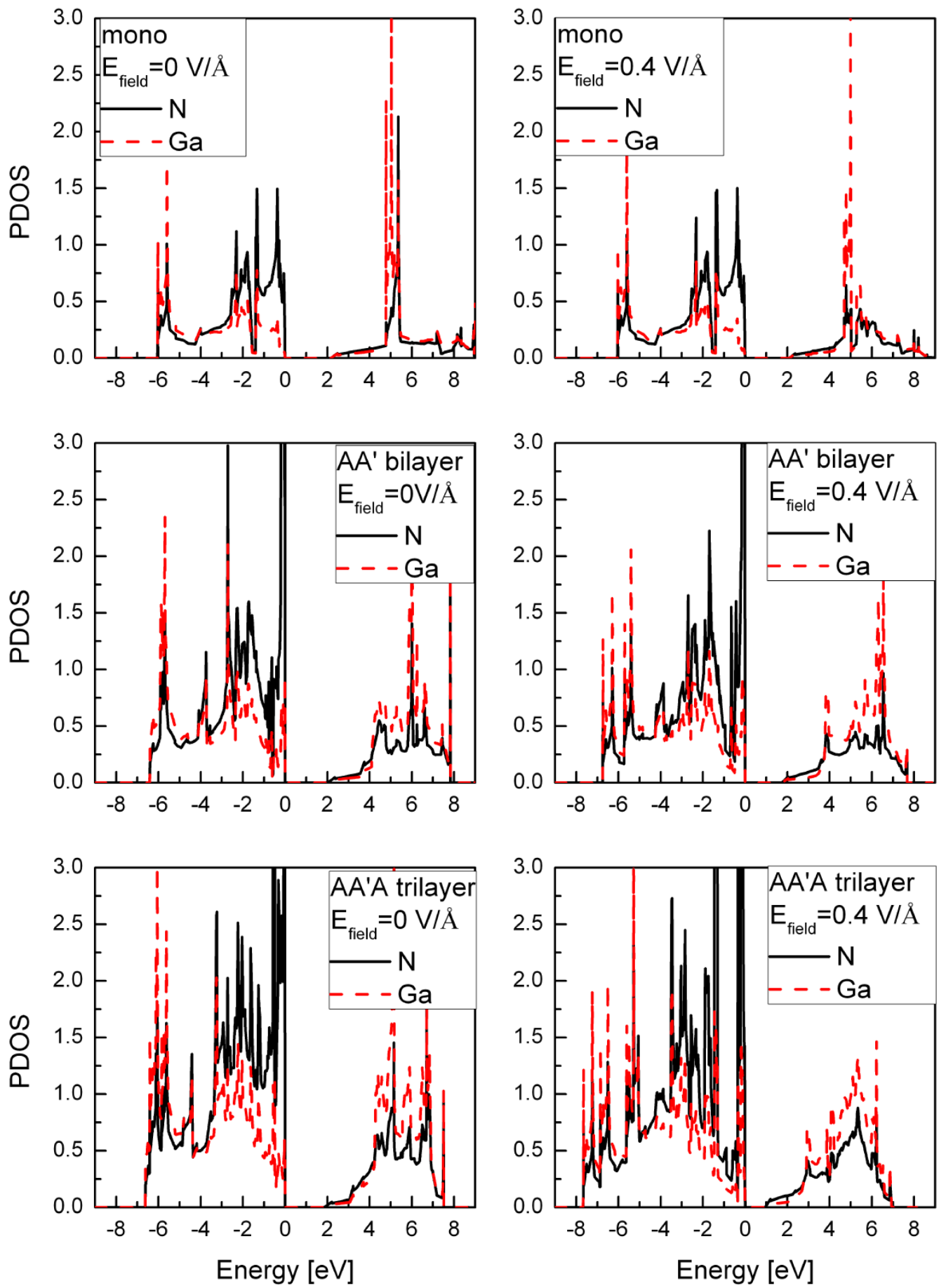

Figure 4. The atom-resolved density of states of the monolayer, the $\mathrm{AA}^{\prime}$-stacked bilayer and the $\mathrm{AA}^{\prime} \mathrm{A}$-stacked trilayer of GaN.

conduction band minimum (CBM), while the energy level of $\mathrm{N}$ shifts down and forms the valence band maximum (VBM) due to the Ga-N interaction (figure 5). For the $\mathrm{AA}^{\prime}$ bilayer, the interlayer interaction between Ga1 (N1) and N2 $(\mathrm{Ga} 2)$ increases the opening of the gap. However, in-plane interaction between $\mathrm{N} 1(\mathrm{~N} 2)$ and $\mathrm{Ga} 1(\mathrm{Ga} 2)$ gets weaker due to increase in the $\mathrm{Ga}-\mathrm{N}$ bond length from $1.85 \AA$ (monolayer) to $1.88 \AA$ (bilayer). At the interlayer level, on the other hand, $\mathrm{N} 1$ (Ga1) is symmetric to $\mathrm{N} 2(\mathrm{Ga} 2)$ resulting in to a degenerate eigenvalues corresponding to the same species of layers. Thus, the degenerate energy levels of Ga1 and Ga2 shifts down forming CBM while those of N1 and N2 shifts up forming the VBM resulting into a smaller band gap for the bilayer as shown in figure 5 .

For the $\mathrm{AA}^{\prime} \mathrm{A}$ stacking, degeneracy between $\mathrm{Ga} 1$ and $\mathrm{Ga} 2$ (N1 and N2) states is removed. Ga2 which interacts with both $\mathrm{N} 1$ and $\mathrm{N} 3$ goes up while Ga1 goes down, resulting in a lowering of the band gap as compared to the $\mathrm{AA}^{\prime}$ stacked bilayer. In addition, the third layer also introduces a new degeneracy between Ga1 and Ga3 (N1 and N3) states (figure 5). 


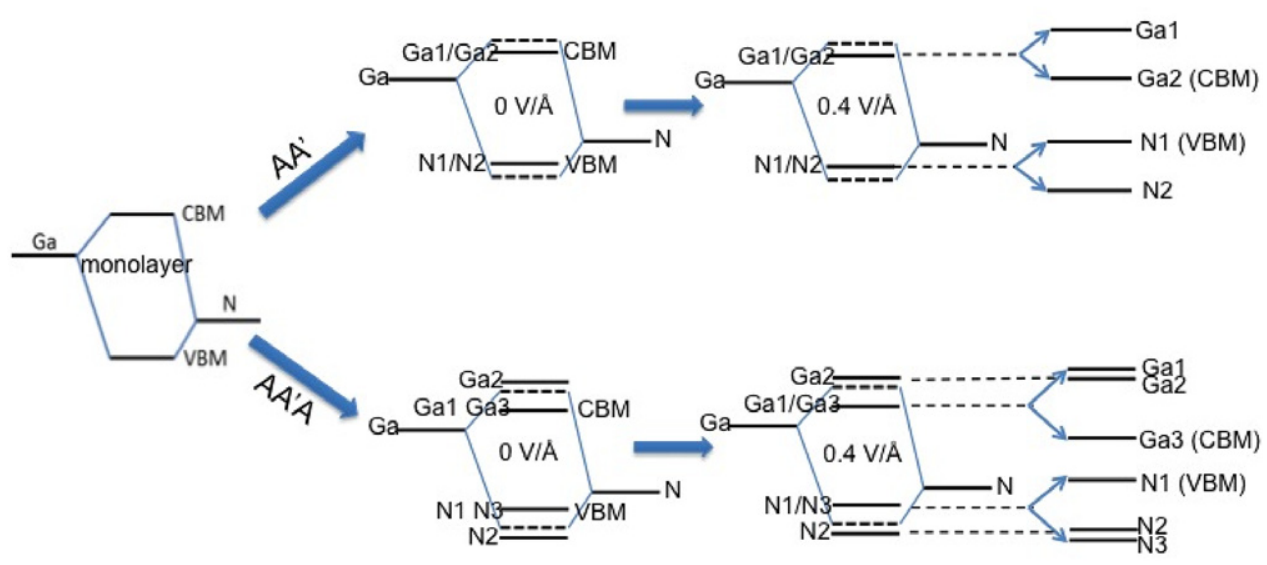

Figure 5. Schematic representation of the orbital interaction model (OIM) of the monolayer, the $\mathrm{AA}^{\prime}$ stacked bilayer and the $\mathrm{AA}^{\prime} \mathrm{A}$ stacked trilayer of GaN.

\subsection{Electronic structure under the applied electric field}

We now investigate the response of the electronic structure of the multilayer $\mathrm{GaN}$ in the presence of an external electric field. We define the electric field from bottom to top to be positive and use the values from -0.4 to $0.4 \mathrm{~V}^{-1}$ for the applied electric field.

In figure 3 the band structure of the multilayer $\mathrm{GaN}$ at $E_{\text {field }}= \pm 0.4 \mathrm{~V}^{-1}$ are shown by dashed lines. For the monolayer, we do not see any noticeable difference in the band structure relative to that obtained at $E_{\text {field }}=0$ (solid lines). For the $\mathrm{AA}^{\prime}$ bilayer, the responses under positive and negative electric fields are symmetric, and the band gap reduces from 2.01 to $1.74 \mathrm{eV}$. Note that the top and bottom $\mathrm{GaN}$ layers exhibit a point symmetry in $\mathrm{AA}^{\prime}$. This is also the case with the trilayer $\mathrm{AA}^{\prime} \mathrm{A}$ where the positive and negative electric field values influence in a similar way because of the symmetry; VBM moves away from $\Gamma$ to $\mathrm{K}$ lowering the band gap from $1.85 \mathrm{eV}\left(E_{\text {field }}=0\right)$ to $0.96 \mathrm{eV}\left(E_{\text {field }}=\right.$ $\pm 0.4 \mathrm{~V}^{-1}$ ). For the $\mathrm{AB}$ (NGa) stacking, almost no changes are seen under applied field strength of $0.4 \mathrm{~V}^{-1}$. In contrast, the application of the field of $\pm 0.4 \mathrm{~V} \AA^{-1}$ drastically reduces the band gap from $1.60 \mathrm{eV}\left(E_{\text {field }}=0 \mathrm{~V}^{-1}\right)$ to $0.33 \mathrm{eV}$ for the $\mathrm{ABA}(\mathrm{NGaN})$-stacked trilayer. As discussed above, there is less electrostatic interlayer interaction in the $\mathrm{ABA}$ stacking as compared to the $\mathrm{AA}^{\prime} \mathrm{A}$ stacking. It is also reflected in the small change of the interplanar separation for the ABA stacking, as compared to the more pronounced monotonic increase of the interplanar separation from 2.49 to $2.52 \AA$ for the $\mathrm{AA}^{\prime} \mathrm{A}$ stacking as the applied field increases from 0 to $0.4 \mathrm{~V}$ (see supplementary information table S1 available at stacks.iop.org/JPhysCM/25/345302/mmedia). This allows a larger polarization across the layers under the external electric field. As a result, the band gap in ABA significantly reduces relative to that in $\mathrm{AA}^{\prime} \mathrm{A}$.

Figure 6 shows the calculated charge density of the layered GaN. Electrons are localized in basins around the $\mathrm{N}$ atoms (the red spots). A clear deficit of electron is seen around $\mathrm{Ga}$ atoms (the blue spots) suggesting the presence of semi-ionic bonding considering the difference in electronegativity of $\mathrm{Ga}$ and N. An overlap is also seen along the vertical direction for the $\mathrm{AA}^{\prime}$ and $\mathrm{AA}^{\prime} \mathrm{A}$ stackings indicating a noticeable $\mathrm{Ga}-\mathrm{N}$ interlayer interaction. A smaller overlap seen for the $\mathrm{AB}(\mathrm{GaN})$ and $\mathrm{ABA}(\mathrm{NGaN})$ stackings suggests the presence of a weaker interlayer interaction in such multilayer GaN.

Figure 7 shows the stacking-dependent variation of the band gap for the multilayer GaN under a perpendicularly applied electric field. A degree of tunability of the band gap gets larger with the increase of the number of layers in the configuration. We would expect this increment to saturate as the number of layers is large enough. Similar band shifting to lower energy under an external electric field was observed for GaN films [41]. For the monolayer, the $\mathrm{AA}^{\prime}$ bilayer and the $\mathrm{AA}^{\prime} \mathrm{A}$ trilayer (left pane), a symmetric variation in the band gap is predicted for the positive and negative applied electric field. For the Bernal stacked layers, the gap tunability shows asymmetric and inconspicuous behavior for the $\mathrm{AB}(\mathrm{GaN})$ stacking. However, variation in the band gap is symmetric and significant for the ABA (NGaN) stacking for which the gap is estimated to close at $E_{\text {field }}= \pm 0.45 \mathrm{~V}^{-1}$. On the other hand, a closing of the band gap is predicted (by extrapolation) at about $E_{\text {field }}= \pm 0.58 \mathrm{~V} \AA^{-1}$ for the $\mathrm{AA}^{\prime} \mathrm{A}$ trilayer. Note that the breakdown field of the bulk GaN is $0.03-0.05 \mathrm{~V}^{-1}$ $\left(3-5 \times 10^{6} \mathrm{~V} \mathrm{~cm}^{-1}\right)[42,43]$. It was previously reported that the breakdown field for ultrathin $\mathrm{BN}\left(0.1 \mathrm{~V} \AA^{-1}\right)$ [44] is larger than that for the hexagonal BN bulk $\left(0.01-0.03 \mathrm{~V}^{-1}\right)$ [45].

Our simplified OIM model (figure 5) provides a reasonable explanation of variation in the band gap with the applied field. Taking bilayer as an example, under a positive bias, both the valence and conduction bands of the top layer shift to a lower energy, while the bands of the bottom layer shift to a higher energy. CBM of $\mathrm{AA}^{\prime}$ primarily consists of electronic states from $\mathrm{Ga} 2$, whereas VBM consists of the N1 states. With the increase of the applied field, the energy levels of $\mathrm{Ga} 2$ and $\mathrm{N} 1$ get closer to each other resulting in the lowering of the band gap. For the $\mathrm{AA}^{\prime} \mathrm{A}$ stacking, degeneracy of the energy levels between the bottom and top layers breaks, 


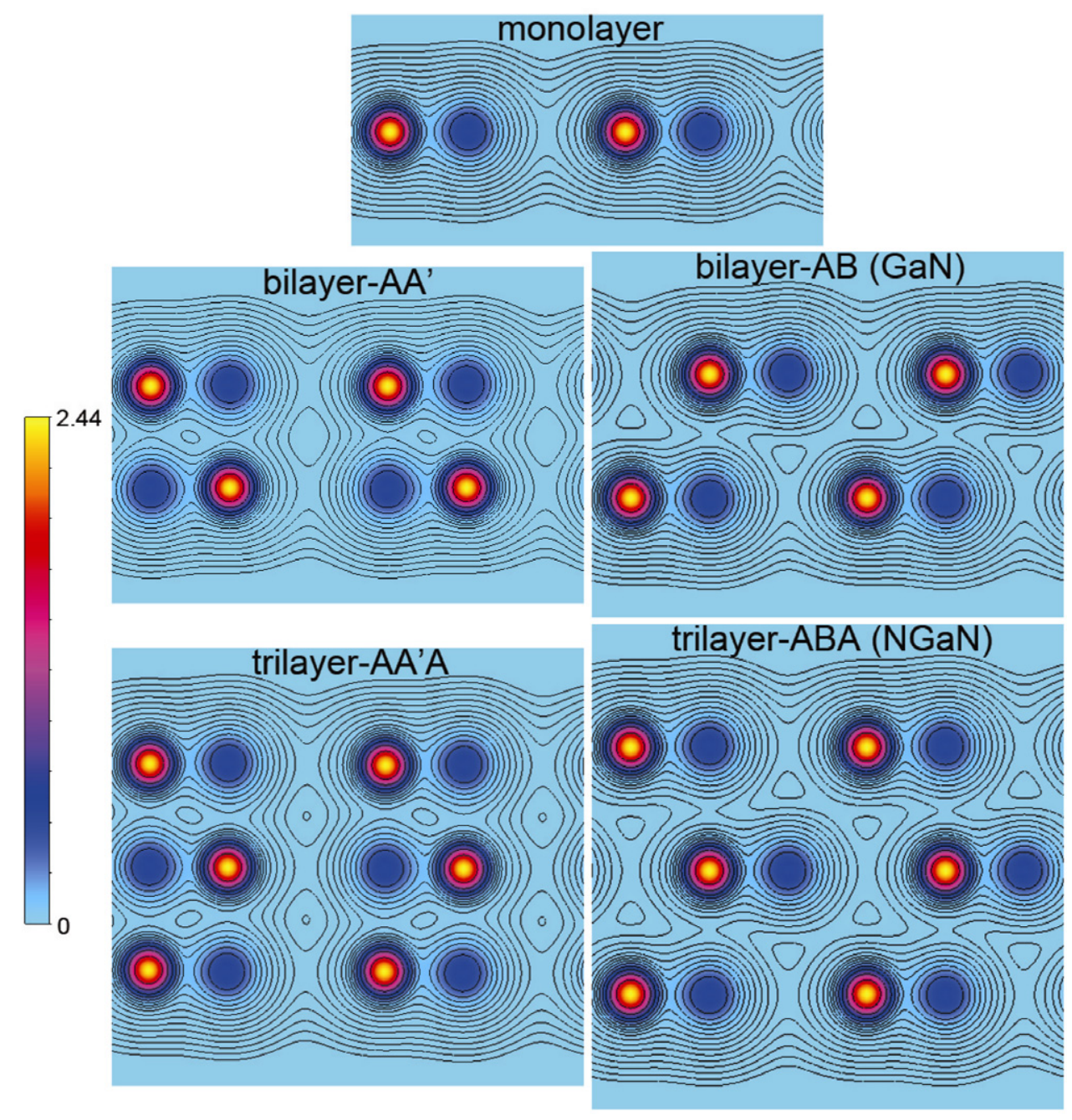

Figure 6. Charge distributions of the monolayer (upper pane), the bilayers (middle pane) and the trilayers (lower pane) of GaN with hexagonal and Bernal stackings. The 2D plane is in the $\langle 1 \overline{1} 0\rangle$ direction. The color scale is labeled in units of $e / \mathrm{bohr}^{3}$.

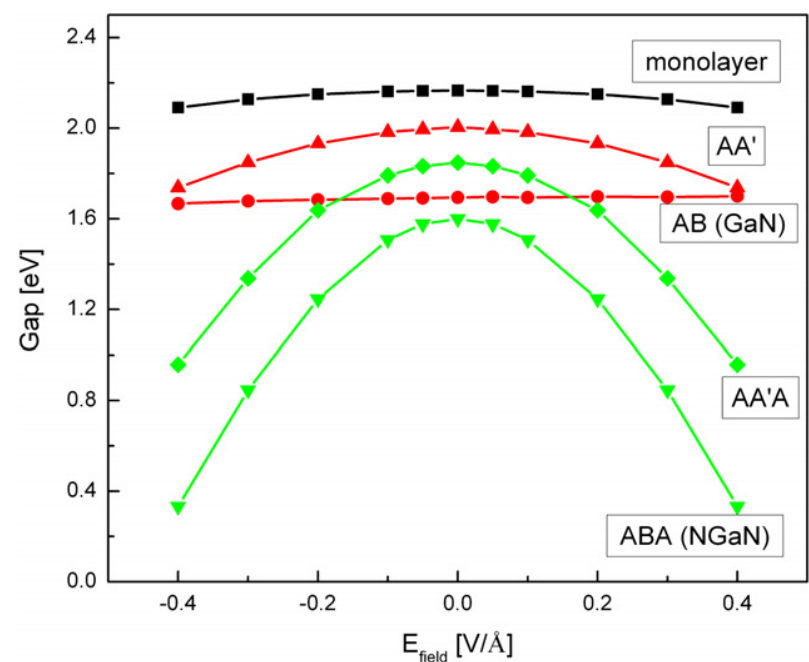

Figure 7. Variation in the band gap with the applied electric field for the multilayer $\mathrm{GaN}$ :monolayer as black squares, $\mathrm{AA}^{\prime}$ bilayer as red up-triangles, $\mathrm{AB}(\mathrm{GaN})$ bilayer as red circles, $\mathrm{AA}^{\prime} \mathrm{A}$ trilayer as green diamonds, and $\mathrm{ABA}(\mathrm{NGaN})$ trilayer as green down-triangles. though the levels of the middle layer are almost unchanged. Using a similar approach as used in the bilayer case, one can derive that the degree of reduction in the band gap is determined by Ga3 and N1 (figure 5). Note that our results on the bilayer $\mathrm{GaN}$ are consistent with the previously reported study on the bilayers of BN [16].

\section{Summary}

The stacking-dependent structural and electronic properties of the atomically thin layers of $\mathrm{GaN}$ are investigated using the $\mathrm{vdW}-\mathrm{DFT}$ level of theory. The $\mathrm{AA}^{\prime}$ and $\mathrm{AA}^{\prime} \mathrm{A}$ stackings are predicted to be the most stable configurations for the bilayer and trilayer of $\mathrm{GaN}$, respectively. A symmetric variation of the band gaps of $\mathrm{AA}^{\prime}$ and $\mathrm{AA}^{\prime} \mathrm{A}$ is predicted with the applied electric field. Overall, the band gap decreases with the increase in the electric field. The proposed OIM model can be used to understand the predicted variation, while a shift in the energy levels is confirmed by analysis of PDOS. It is notable that the band gap can be varied by changing the stacking orders of the multilayer GaN. A response to an 
external electric field applied perpendicular to the layer is the largest for the ABA (NGaN) trilayer. Furthermore, the calculated results predict tuning of the band gap from 1.6 to $0.33 \mathrm{eV}$ with $E_{\text {field }}$ varying from 0 to $\pm 0.4 \mathrm{~V}^{-1}$.

It is expected that the calculated results may provide a fundamental basis for applications of the multilayer $\mathrm{GaN}$ as a candidate semiconducting material for novel optoelectronic devices. With great advances recently made in fabrication of $\mathrm{GaN}$ based nanostructures, e.g. nanorods [46, 47], nanowires [48, 49], nanosaws [50] and nanotubes [51], an accurate control of the stacking sequence and the thickness of the multilayer GaN structures may soon become possible. A detailed understanding of these low-dimensional building blocks of $\mathrm{GaN}$ may also help us in developing nanoscale optoelectronic devices utilizing the advanced modern fabrication techniques.

\section{Acknowledgments}

Helpful discussions with Professor Jun $\mathrm{Ni}$ and Xiaoliang Zhong are acknowledged. The work at Michigan Technological University was performed under support by the Army Research Laboratory through Contract Number W911NF-09-2-0026.

\section{References}

[1] Matovic J and Jakšić Z 2009 Nanomembrane: a new MEMS/NEMS building block Micro Electronic and Mechanical Systems ed Kenichi Takahata (Rijeka: InTech)

[2] Rogers J A, Lagally M G and Nuzzo R G 2011 Synthesis, assembly and applications of semiconductor nanomembranes Nature 477 45-53

[3] Cavallo F and Lagally M G 2012 Semiconductor nanomembranes: a platform for new properties via strain engineering Nanoscale Res. Lett. 7 1-10

[4] Nakamura S, Pearton S and Fasol G 2000 The Blue Laser Diode: The Complete Story (Berlin: Springer)

[5] Krames M R et al 2007 Status and future of high-power light-emitting diodes for solid-state lighting J. Disp. Technol. 3 160-75

[6] Mishra U K et al 2008 GaN-based RF power devices and amplifiers Proc. IEEE 96 287-305

[7] Maruska H P and Tietjen J J 1969 The preparation and properties of vapor-deposited single-crystal-line GaN Appl. Phys. Lett. 15 327-9

[8] Ponce F A and Bour D P 1997 Nitride-based semiconductors for blue and green light-emitting devices Nature 386 351-9

[9] Joblot $\mathrm{S}$ et al 2005 High-electron-mobility AlGaN/GaN heterostructures grown on $\mathrm{Si}(001)$ by molecular-beam epitaxy Appl. Phys. Lett. 87133505

[10] Zhang Y, Leung B and Han J 2012 A liftoff process of GaN layers and devices through nanoporous transformation Appl. Phys. Lett. 100181908

[11] Tiginyanu I, Popa V and Stevens-Kalceff M A 2011 Ultra-thin GaN membranes fabricated by using surface charge lithography ECS Trans. 35 13-9

[12] Takagaki Y et al 2004 Strain relaxation in AlN/GaN bilayer films grown on gamma- $\mathrm{LiAlO}_{2}(100)$ for nanoelectromechanical systems Appl. Phys. Lett. 84 4756-8

[13] Mei Y et al 2009 Fabrication, self-assembly, and properties of ultrathin $\mathrm{AlN} / \mathrm{GaN}$ porous crystalline nanomembranes: tubes, spirals, and curved sheets ACS Nano 3 1663-8
[14] Chen Q A et al 2011 Tailoring band gap in GaN sheet by chemical modification and electric field: $a b$ initio calculations Appl. Phys. Lett. 98053102

[15] Amorim R G et al 2013 Strain- and electric field-induced band gap modulation in nitride nanomembranes $J$. Phys.: Condens. Matter 25195801

[16] Yang Z and Ni J 2010 Modulation of electronic properties of hexagonal boron nitride bilayers by an electric field: a first principles study J. Appl. Phys. 107104301

[17] Zhong X et al 2012 Electronic structure and quantum transport properties of trilayers formed from graphene and boron nitride Nanoscale 4 5490-8

[18] Ramasubramaniam A, Naveh D and Towe E 2011 Tunable band gaps in bilayer graphene-BN heterostructures Nano Lett. 11 1070-5

[19] Min $\mathrm{H}$ et al 2007 Ab initio theory of gate induced gaps in graphene bilayers Phys. Rev. B 75155115

[20] Lui C H et al 2011 Observation of an electrically tunable band gap in trilayer graphene Nature Phys. 7 944-7

[21] Balu R et al 2012 Effect of electric field on the band structure of graphene/boron nitride and boron nitride/boron nitride bilayers Appl. Phys. Lett. 100052104

[22] Kresse G and Furthmüller J 1996 Efficient iterative schemes for $a b$ initio total-energy calculations using a plane-wave basis set Phys. Rev. B 54 11169-86

[23] Kresse G and Joubert D 1999 From ultrasoft pseudopotentials to the projector augmented-wave method Phys. Rev. B 59 1758-75

[24] Perdew J P, Burke K and Ernzerhof M 1996 Generalized gradient approximation made simple Phys. Rev. Lett. $773865-8$

[25] Grimme S 2006 Semiempirical GGA-type density functional constructed with a long-range dispersion correction J. Comput. Chem. 27 1787-99

[26] Costales A et al 2002 Chemical bonding in group III nitrides J. Am. Chem. Soc. 124 4116-23

[27] Pandey R, Jaffe J E and Harrison N M 1994 Ab-initio study of high-pressure phase-transition in GaN J. Phys. Chem. Solids 55 1357-61

[28] Paszkowicz W, Podsiadło S and Minikayev R 2004 Rietveld-refinement study of aluminium and gallium nitrides J. Alloys Compounds 382 100-6

[29] Stampfl C and Van de Walle C G 1999 Density-functional calculations for III-V nitrides using the local-density approximation and the generalized gradient approximation Phys. Rev. B 59 5521-35

[30] Lee S M et al 1999 Stability and electronic structure of GaN nanotubes from density-functional calculations Phys. Rev B 60 7788-91

[31] Sahin $\mathrm{H}$ et al 2009 Monolayer honeycomb structures of group-IV elements and III-V binary compounds: first-principles calculations Phys. Rev. B 80155453

[32] Zoroddu A et al 2001 First-principles prediction of structure, energetics, formation enthalpy, elastic constants, polarization, and piezoelectric constants of $\mathrm{AlN}, \mathrm{GaN}$, and InN: comparison of local and gradient-corrected density-functional theory Phys. Rev. B 64045208

[33] de Andres P L, Ramírez R and Vergés J A 2008 Strong covalent bonding between two graphene layers Phys. Rev. B 77045403

[34] Zhong X et al 2011 First-principles study of strain-induced modulation of energy gaps of graphene/BN and $\mathrm{BN}$ bilayers Phys. Rev. B 83193403

[35] Yuan S, Roldán R and Katsnelson M I 2011 Landau level spectrum of ABA- and ABC-stacked trilayer graphene Phys. Rev. B 84125455

[36] Castro Neto A H et al 2009 The electronic properties of graphene Rev. Mod. Phys. 81 109-62 
[37] Zou K et al 2013 Transport studies of dual-gated ABC and $\mathrm{ABA}$ trilayer graphene: band gap opening and band structure tuning in very large perpendicular electric fields Nano Lett. 13 369-73

[38] Li $\mathrm{H}$ et al 2010 Electronic structures and magnetic properties of GaN sheets and nanoribbons J. Phys. Chem. C 114 11390-4

[39] Li H et al 2012 Layer number-dependent structural evolution of two-dimensional diamond films Chem. Phys. Lett. 550 130-3

[40] Marom N et al 2010 Stacking and registry effects in layered materials: the case of hexagonal boron nitride Phys. Rev. Lett. 105046801

[41] Jeong T S et al 2005 Electric-field-induced quenching effect of photoluminescence on p-GaN films J. Korean Phys. Soc 46 968-72

[42] Levinshtein M E, Rumyantsev S L and Shur M S (ed) 1999 Handbook Series on Semiconductor Parameters vols 1 and 2 (London: World Scientific)

[43] Chow T P and Ghezzo M J 1996 SiC Power Devices MRS Proceedings 423 9-21
[44] Britnell L et al 2012 Electron tunneling through ultrathin boron nitride crystalline barriers Nano Lett. 12 1707-10

[45] Edgar J H (ed) 1994 Properties of Group III Nitrides, Electronic Materials Information Service (EMIS) (London: INSPEC, Institution of Electrical Engineers)

[46] Li F et al 2010 UV photovoltaic cells fabricated utilizing GaN nanorod/Si heterostructures J. Cryst. Growth 312 2320-3

[47] Park C M et al 2006 Optical properties of GaN nanorods grown by molecular-beam epitaxy; dependence on growth time Nanotechnology 17952

[48] Hersee S D, Sun X and Wang X 2006 The controlled growth of GaN nanowires Nano Lett. 6 1808-11

[49] Kuykendall T et al 2004 Crystallographic alignment of high-density gallium nitride nanowire arrays Nature Mater. 3 524-8

[50] Bae S Y et al 2003 Large-scale synthesis of gallium nitride nanosaws using a chemical vapor deposition method Chem. Phys. Lett. 373 620-5

[51] Goldberger J et al 2003 Single-crystal gallium nitride nanotubes Nature 422 599-602 